\title{
Arrion Cobertura vacinal em crianças de até 2 anos de idade beneficiárias do Programa Bolsa Família, Brasil*
}

doi: 10.1590/S1679-49742021000300010

\author{
Vaccination coverage in children up to 2 years old, receiving financial support from the Family \\ Income Transfer Program, Brazil
}
Cobertura de vacunación de niños hasta 2 años, beneficiarios del Programa Bolsa Família, Brasil

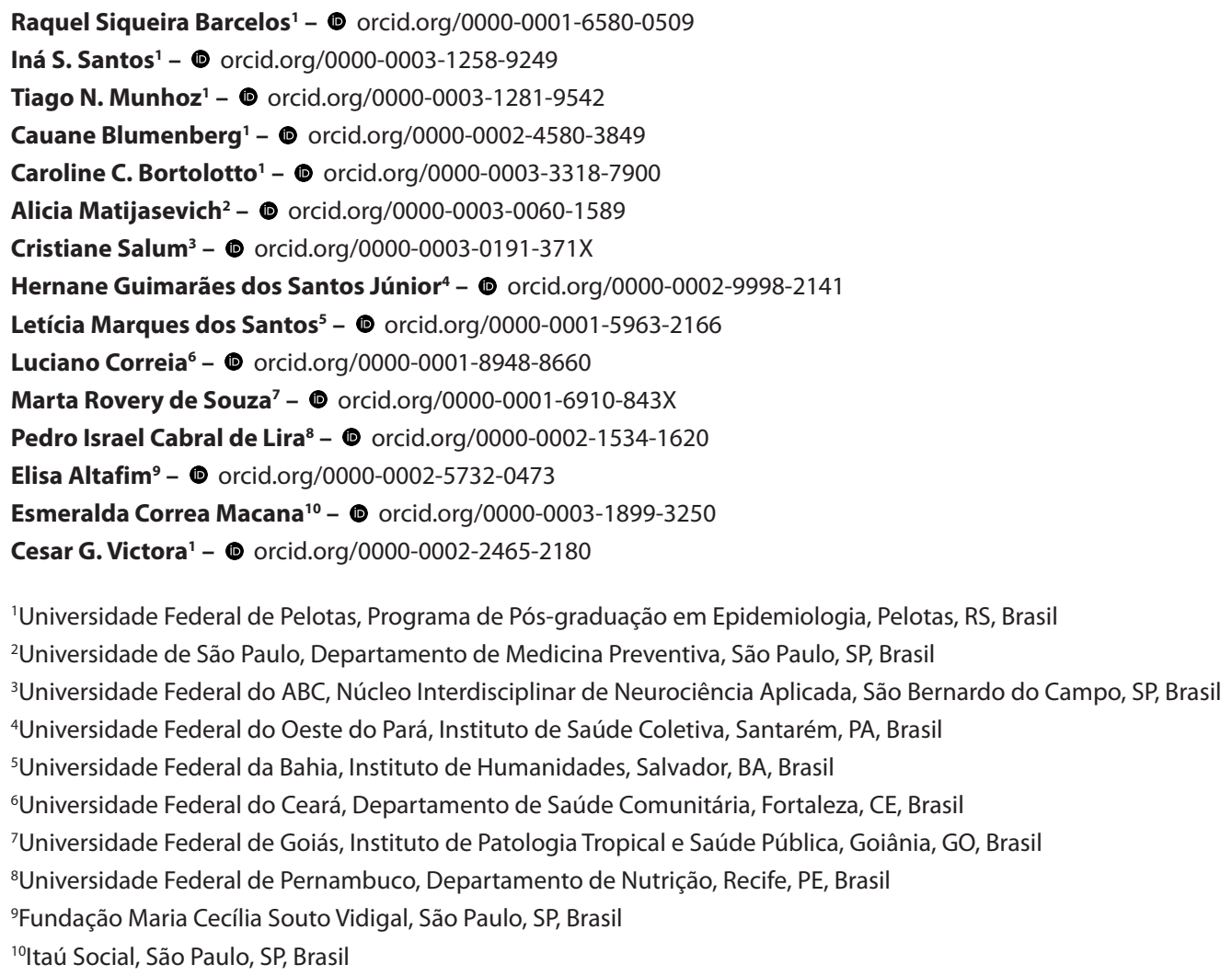

*Estudo financiado pela Fundação Maria Cecília Souto Vidigal (FMCSV), Itaú Social e Ministério da Cidadania, com o apoio do Programa das Nações Unidas para o Desenvolvimento (PNUD) (BRA/16/019). Santos IS, Matijasevich A, Lira PIC e Victora CG recebem bolsas de produtividade em pesquisa concedidas pelo Conselho Nacional de Desenvolvimento Científico e Tecnológico (CNPq)/Ministério da Ciência, Tecnologia, Inovações e Comunicações (MCTIC). Bortolotto CC conta com apoio financeiro da Coordenação de Aperfeiçoamento de Pessoal de Nível Superior (Capes)/Ministério da Educação (MEC) (código 001).

\section{Endereço para correspondência:}

Raquel Siqueira Barcelos - Universidade Federal de Pelotas, Programa de Pós-Graduação em Epidemiologia, Rua Marechal Deodoro, no 1160, $3^{\circ}$ andar, Pelotas, RS, Brasil. CEP: 96020-220

E-mail: bio.raquelbarcelos@gmail.com 


\section{Resumo}

Objetivo: Avaliar a cobertura vacinal, conforme o calendário do Programa Nacional de Imunizações, entre crianças beneficiárias do Programa Bolsa Família, Brasil, segundo nível socioeconômico da família e características maternas. Métodos: Foram avaliadas 3.242 crianças menores de 12 meses de vida entre agosto de 2018 e abril de 2019, sendo 3.008 delas reavaliadas entre setembro de 2019 e janeiro de 2020. As análises foram realizadas utilizando-se modelos multiníveis (nível 3, Unidade da Federação; nível 2, município; nível 1, crianças). Resultados: A cobertura vacinal foi 2,5 vezes maior no primeiro $\left(61,0 \%-\mathrm{IC}_{95 \%} 59,3 ; 62,6 \%\right)$, comparado ao segundo acompanhamento $\left(24,8 \%-\mathrm{IC}_{95 \%} 22,8 ; 25,9 \%\right)(\mathrm{p}<0,001)$. No primeiro acompanhamento, a cobertura foi maior no quintil mais rico $(67,9 \%)$ e entre as crianças cujas mães tinham $\geq 9$ anos de escolaridade (63,3\%). No segundo acompanhamento, não houve diferenças. As maiores coberturas ocorreram entre 0,5-2,5 (93,5\%) e 12,5-15,5 meses (34,4\%), respectivamente primeiro e segundo acompanhamentos. Conclusão: Encontrou-se baixa cobertura, tanto no primeiro quanto no segundo ano de vida.

Palavras-chave: Cobertura Vacinal; Criança; Imunização; Estudos Longitudinais.

\section{Introdução}

Vacinas estão entre as medidas de Saúde Pública mais efetivas para prevenção, eliminação e controle de doenças transmissíveis. ${ }^{1}$ 0s programas organizados de imunização são, reconhecidamente, um dos investimentos de maior custo-efetividade em saúde. ${ }^{2}$ No Brasil, o Programa Nacional de Imunizações (PNI), criado em 1973, de acesso universal e contínuo, oferece amplo leque de vacinas, disponibilizadas nos serviços públicos de saúde de todo o território nacional, sem ônus para os usuários. Ademais, a cada ano, o PNI promove várias campanhas de vacinação em massa. ${ }^{3} \mathrm{~A}$ oferta universal de vacinas na rede pública e seu acesso facilitado à população, dada a extensa rede nacional de serviços básicos de saúde, juntamente com outras ações de vigilância, têm sido fundamentais para a eliminação e controle de doenças como varíola, difteria, poliomielite e sarampo, historicamente responsáveis por um grande número de vítimas no Brasil. ${ }^{4}$

\section{Vacinas estão entre as medidas de Saúde Pública mais efetivas para prevenção, eliminação e controle de doenças transmissíveis.}

Atualmente, 0 PNI oferece 44 tipos de imunobiológicos, incluindo vacinas, soros e imunoglobulinas. ${ }^{3,4}$ No entanto, o aumento na complexidade de itens do calendário vacinal, nas últimas décadas, e a introdução de diversas vacinas em um curto período, trouxeram novos desafios para programa, entre eles o de atingir e manter altas coberturas vacinais na população. ${ }^{5}$ Apenas para o primeiro ano de vida, por exemplo, o calendário atual de vacinação prevê a realização de oito contatos entre a família e os serviços de saúde, para que a criança receba um total de 18 doses de vacinas. Não obstante, pesquisas realizadas em locais específicos do país sinalizaram uma possível redução na cobertura vacinal, no período de 1993 a $2015 .^{6}$

0 objetivo deste estudo foi avaliar a cobertura vacinal, conforme o calendário do PNI, entre crianças beneficiárias do Programa Bolsa Família, de acordo com 0 nível socioeconômico da família e características maternas.

\section{Métodos}

Trata-se da primeira e segunda etapas do estudo longitudinal 'Avaliação de Impacto do Programa Criança Feliz (PCF)', oferecido pelo Ministério da Cidadania (MCid) a menores de 3 anos de idade 7 , com o objetivo de promover o desenvolvimento infantil por meio do fortalecimento dos vínculos familiares, prevenção de situações de negligência e violência contra a criança e redução da desnutrição.

A população-alvo do PCF constitui-se de crianças beneficiárias do Bolsa Família, ${ }^{8}$ cuja participação no programa é condicionada ao cumprimento, pela família, de algumas exigências: matricular e manter as crianças e adolescentes de 6 a 17 anos na escola; $\mathrm{e}$ levar os menores de 7 anos a unidades de saúde, para imunização e monitoração do crescimento e desenvolvimento, conforme calendário recomendado pelas equipes de saúde. 
Desde 2018, está sendo realizado o trabalho 'Avaliação de Impacto do Programa Criança Feliz', um estudo randomizado, cujo objetivo é estimar o efeito do programa na estimulação intelectual do ambiente doméstico e no neurodesenvolvimento das crianças atendidas pelo Bolsa Família, antes de completarem 0 primeiro ano de vida. ${ }^{9}$ Para compor o estudo, foram escolhidas seis Unidades da Federação (UFs), com grande clientela do Bolsa Família. Em cada UF, foram selecionados três a seis municípios com sobredemanda (no mínimo 4:1) de menores de 1 ano de idade elegíveis para o PCF; e que contassem com uma capacidade instalada para incluir e visitar semanalmente $\geq 80$ crianças. Ao todo, foram selecionados 30 municípios dos estados da Bahia, Ceará, Goiás, Pará, Pernambuco e São Paulo (média de 109 crianças por município). Mais informações sobre a metodologia adotada no estudo estão disponíveis em: https://aplicacoes.mds. gov.br/sagirmps/ferramentas/docs/Caderno\%20de\%20 Estudos-35-online.pdf; e http://www.epidemio-ufpel. org.br/uploads/downloads/avaliacao-do-impacto-doprograma-crianca-feliz.pdf.

0 MCid fornecia à equipe estadual uma lista de crianças e gestantes elegíveis para o PCF. Chegando a cada município, a equipe de avaliação reunia-se com 0 responsável pelo PCF no Centro de Referência de Assistência Social (CRAS), para delimitar os bairros onde 0 programa de avaliação seria disponibilizado. Na maioria dos municípios, o PCF não é oferecido em áreas rurais, áreas urbanas remotas ou onde há problemas de segurança. De posse da lista fornecida pelo MCid à equipe estadual do PCF, os pesquisadores visitavam cada domicílio para determinar se a família aceitaria participar do programa e da pesquisa. A inclusão de gestantes deveu-se à possibilidade de essas mulheres haverem dado à luz após a última atualização da listagem, que, normalmente, correspondia a alguns meses antes do trabalho de coleta de dados (Material Suplementar 4).

No atual estudo, foram analisadas as 3.242 crianças avaliadas no primeiro acompanhamento (linha de base - T0), ocorrido entre agosto de 2018 e julho de 2019, quando estavam com menos de 12 meses de vida; e as 3.008 crianças ( $93 \%$ do grupo original) localizadas e avaliadas no segundo acompanhamento (T1), ocorrido 9-13 meses após o início do T0 (mediana=12 meses), conforme a UF.
Os questionários utilizados na coleta de dados, em ambas as etapas, foram testados previamente, em estudo-piloto realizado na cidade de Pelotas, RS, e estão disponíveis no sítio eletrônico do Programa de Pós-Graduação em Epidemiologia da Universidade Federal de Pelotas. ${ }^{10}$

Nas duas avaliações - T0 e T1 -, o questionário geral, aplicado por entrevistadores treinados, continha perguntas já testadas e padronizadas, extraídas do Multiple Indicator Cluster Survey (MICS) ${ }^{11}$ dos instrumentos utilizados nas Coortes de Nascimento de Pelotas de 2004 e $2015^{12,13}$ e dos manuais de treinamento do PCF. ${ }^{14}$ Os entrevistadores foram selecionados após participar de curso de treinamento, com duração de 40 horas, realizado em Brasília, DF. As respostas às perguntas eram registradas em tablets, sobre plataforma REDCap. ${ }^{15}$

Em ambas as visitas, era solicitado à mãe que mostrasse a Caderneta de Saúde da Criança ou qualquer outro registro de vacinação. Também com 0 auxílio do tablet, o entrevistador fotografava e gravava a imagem da Caderneta; em seguida, registrava as doses de vacinas já recebidas pela criança. Para cada vacina, havia cinco opções de preenchimento da resposta, de múltipla escolha: ' 1 a dose'; ' 2 a dose'; ' 3 a dose'; 'não fez'; e 'reforços'. Caso a criança tivesse recebido a primeira e a segunda doses de uma vacina, eram marcadas as opções ' 1 a dose' e ' 2 a dose'.

Para as análises, o desfecho 'vacinação adequada' foi operacionalizado conforme o calendário vacinal recomendado pelo $\mathrm{PNI}^{16} \mathrm{e}$ esquematizado na Figura 1 . As crianças que tivessem recebido todas as vacinas recomendadas para sua idade (observada uma tolerância de até 15 dias de atraso para cada imunobiológico) eram consideradas com vacinação 'adequada'. Por exemplo, uma criança com 6 meses e 7 dias de vida era classificada como 'em dia' com o calendário se tivesse recebido todas as vacinas previstas para os 5 meses de vida, mesmo que alguma dose anterior tivesse sido administrada com algum atraso. Vacinas que não compõem o esquema básico de vacinação do $\mathrm{PNI}^{16}$ (como a da influenza), bem como aquelas não oferecidas na rotina dos serviços em todas as UFs (e.g., febre amarela), ${ }^{17}$ não entraram na construção da variável de desfecho.

As variáveis independentes utilizadas foram coletadas na entrevista do T0: 
a) Nível socioeconômico da família (dividido em quintis, e construído a partir da informação sobre características do domicílio e bens de consumo, mediante análise de componentes principais);

b) Idade materna (anos: $<20 ; 20-29$; 30-39; $\geq 40$ );

c) Escolaridade materna (anos de estudo: 0-4;5-8;

$\geq 9$ );

d) Raça/cor da pele autodeclarada da mãe (bran-

ca; parda [reuniu as categorias morena/parda, amarela/asiática e indígena]; e preta);

e) Vive com marido/companheiro (sim; não).

Como a cobertura vacinal no T1 foi diferente no grupo PCF e no grupo de controle $(\mathrm{p}=0,032)$, utilizou-se o status 'intervenção' ou o status 'controle' das crianças como potencial confundidor da associação entre as exposições e os desfechos.

A alocação aleatória dos participantes para 0 grupo de controle ou para o grupo de intervenção e a semelhança entre os grupos no T0 e em relação às perdas podem ter prevenido viés de seleção. 0 viés de informação foi minimizado com a padronização da coleta de dados: as equipes das seis UFs foram treinadas na mesma ocasião, e a informação sobre as vacinas, obtida diretamente da Carteira de Saúde da Criança.

0 tamanho da amostra necessário para este estudo de avaliação do impacto do PCF foi calculado com base nos seguintes parâmetros: erro alfa bicaudal de 5\%; erro beta de $10 \%$; adesão ao programa de $60 \%$ (continuidade de participação no PCF durante três anos); e um acréscimo de $20 \%$ para perdas de acompanhamento. Dessa forma, seria necessário consultar os dados de 2.880 crianças do país.

As análises estatísticas foram realizadas com uso do programa Stata versão 16.0 (StataCorp LLC, College Station, TX, Estados Unidos). A proporção de crianças com Caderneta de Saúde ou outro registro de vacinação foi a primeira variável a ser calculada. Posteriormente, estimou-se a proporção de crianças com vacinação adequada no T0 e T1, para toda a amostra, com os respectivos intervalos de confiança de $95 \%\left(\mathrm{IC}_{95 \%}\right)$. Logo, foram calculadas as proporções de crianças com vacinação adequada, conforme as características da família e maternas. Em seguida, foram calculadas as proporções por UF e município. Nos dois acompanhamentos (T0 e T1), foram utilizados modelos multiníveis (nível 3, UF; nível 2, município; nível 1, crianças). As associações foram avaliadas pelo teste de
Wald, sendo os valores $\mathrm{p}<0,05$ considerados estatisticamente significantes.

0 projeto do estudo foi submetido ao Comitê de Ética em Pesquisa da Faculdade de Medicina da Universidade Federal de Pelotas (CEP/FAMED/UFPel), afiliado à Comissão Nacional de Ética em Pesquisa (Conep)/Conselho Nacional de Saúde (CNS), e aprovado no Parecer CNS no 2.148.689, emitido em 13 de maio de 2017. 0 projeto está disponível para consulta no sítio eletrônico do Registro Brasileiro de Ensaios Clínicos (ReBEC), sob o identificador RBR-4x7dny. 0 Termo de Consentimento Livre e Esclarecido foi assinado pelas mães ou responsáveis, como condição para participar do estudo.

\section{Resultados}

As listagens fornecidas pelo MCid para os $30 \mathrm{mu}-$ nicípios apresentavam 8.601 famílias com gestantes ou crianças potencialmente elegíveis para inclusão no PCF. Das 8.601 famílias listadas, mais da metade não foi incluída no estudo porque 0 endereço não foi localizado pelos pesquisadores (28\%), a família havia se mudado definitivamente (15\%) ou estava temporariamente ausente do domicílio (13,3\%) (Material Suplementar 4). Ao todo, 3.242 (37,7\%) das famílias listadas pelo MCid foram efetivamente contempladas na pesquisa.

Entre as 3.242 crianças avaliadas no $\mathrm{T} 0$, a mediana de idade foi de 7,6 meses; para as 3.008 reavaliadas no T1, a mediana de idade foi de 18,9 meses. Na Tabela 1, observa-se a distribuição da amostra de acordo com as características na linha de base (T0). A maior proporção de mães encontrava-se entre 20-29 anos de idade $(51,5 \%)$, tinham $\geq 9$ anos de escolaridade $(60,0 \%), 75,4 \%$ autodeclararam-se de raça/cor da pele parda e mais de $60 \%$ informaram viver com marido ou companheiro.

No T1, houve perda de 234 crianças (de 7,2\% em relação ao T0). Entre as crianças não acompanhadas no T1, a maior proporção pertencia ao quintil mais pobre $(23,5 \%)$, as mães tinham entre 20 e 29 anos de idade $(55,2 \%)$, menos de nove anos de estudo (52,1\%), autodeclararam-se de raça/cor da pele parda $(73,2 \%)$ e viviam com marido/companheiro (66,4\%).

Um total de 3.133 (96,7\%) famílias no T0 e 2.779 $(92,4 \%)$ no T1 apresentaram a Caderneta de Saúde da 


\begin{tabular}{|c|c|c|}
\hline Grupo de idade & Idade recomendada (meses) & Vacinas recomendadas \\
\hline \multirow{2}{*}{1} & \multirow{2}{*}{$0 \leq$ idade $<2$} & $B C G^{a}$ \\
\hline & & Hepatite B (pelo menos 1 dose) \\
\hline \multirow{5}{*}{2} & \multirow{5}{*}{$2 \leq$ idade $<3$} & Vacinas do grupo de idade 1 \\
\hline & & Pentavalente \\
\hline & & VII $\mathrm{P}^{\mathrm{b}}$ \\
\hline & & Pneumocócica 10-valente \\
\hline & & Rotavírus humano \\
\hline \multirow{3}{*}{3} & \multirow{3}{*}{$3 \leq$ idade $<4$} & Vacinas do grupo de idade 1 \\
\hline & & Vacinas do grupo de idade 2 \\
\hline & & Meningocócica C (pelo menos 1 dose) \\
\hline \multirow{6}{*}{4} & \multirow{6}{*}{$4 \leq$ idade $<5$} & Vacinas do grupo de idade 1 \\
\hline & & Pentavalente (pelo menos 2 doses) \\
\hline & & VIPb (pelo menos 2 doses) \\
\hline & & Pneumocócica 10-valente (pelo menos 2 doses) \\
\hline & & Rotavírus humano (pelo menos 2 doses) \\
\hline & & Meningocócica C (pelo menos 1 dose) \\
\hline \multirow{6}{*}{5} & \multirow{6}{*}{$5 \leq$ idade $<6$} & Vacinas do grupo de idade 1 \\
\hline & & Pentavalente (pelo menos 2 doses) \\
\hline & & VIP (pelo menos 2 doses) \\
\hline & & Pneumocócica 10-valente (pelo menos 2 doses) \\
\hline & & Rotavírus humano (pelo menos 2 doses) \\
\hline & & Meningocócica ( (pelo menos 2 doses) \\
\hline \multirow{6}{*}{6} & \multirow{6}{*}{$6 \leq$ idade $<9$} & Vacinas do grupo de idade 1 \\
\hline & & Pentavalente (pelo menos 3 doses) \\
\hline & & VIPb (pelo menos 3 doses) \\
\hline & & Pneumocócica 10-valente (pelo menos 2 doses) \\
\hline & & Rotavírus humano (pelo menos 2 doses) \\
\hline & & Meningocócica ( (pelo menos 2 doses) \\
\hline \multirow{2}{*}{7} & \multirow{2}{*}{$9 \leq$ idade $<12$} & Vacinas do grupo de idade 1 \\
\hline & & Vacinas do grupo de idade 6 \\
\hline \multirow{7}{*}{8} & \multirow{7}{*}{$12 \leq$ idade $<15$} & Vacinas do grupo de idade 1 \\
\hline & & Pentavalente (pelo menos 3 doses) \\
\hline & & VIPb (pelo menos 3 doses) \\
\hline & & Pneumocócica 10-valente (pelo menos 3 doses) \\
\hline & & Rotavírus humano (pelo menos 2 doses) \\
\hline & & Meningocócica ( (pelo menos 3 doses) \\
\hline & & Tríplice viral (pelo menos 1 dose) \\
\hline
\end{tabular}


Continuação

\begin{tabular}{|l|l|l|}
\hline Grupo de idade & Idade recomendada (meses) & Vacinas recomendadas \\
\hline \multirow{5}{*}{9} & \multirow{4}{*}{$15 \leq$ idade $<48$} & Vacinas do grupo de idade 1 \\
\cline { 3 - 3 } & & Vacinas do grupo de idade 8 \\
\cline { 3 - 3 } & & DTP( reforço (pelo menos 1 dose) \\
\cline { 3 - 3 } & Vopd reforço (pelo menos 1 dose) \\
\cline { 3 - 3 } & Hepatite A \\
\cline { 3 - 3 } & Tetraviral \\
\hline
\end{tabular}

a) BCG: bacilo de (almette-Guérin; b) VIP: poliomielite 1,2,3; c) DTP: vacina adsorvida difteria, tétano e pertussis; d) VOP: poliomielite 1 e 3.

Figura 1 - Esquema vacinal adotado para a construção da variável 'vacinação adequada' conforme calendário vacinal, extraído e adaptado do Calendário de Vacinação da Caderneta de Saúde da Criança

Tabela 1 - Características familiares e maternas na linha de base (T0) do estudo Avaliação do Impacto do Programa Criança Feliz ( $n=3.242$ crianças $<12$ meses de vida), Brasil, agosto/2018-abril/2019

\begin{tabular}{|c|c|c|}
\hline Características familiares e maternas & N & $\%$ \\
\hline \multicolumn{3}{|c|}{ Nível socioeconômico da família (quintis) (n=3.239) } \\
\hline $10^{\circ}$ (mais pobre) & 648 & 20,0 \\
\hline $2^{0}$ & 648 & 20,0 \\
\hline $3^{\circ}$ & 648 & 20,0 \\
\hline $4^{0}$ & 650 & 20,1 \\
\hline $5^{0}$ (mais rico) & 645 & 19,9 \\
\hline \multicolumn{3}{|l|}{ Idade materna (anos) $(\mathrm{n}=3.199)$} \\
\hline$<20$ & 464 & 14,5 \\
\hline 20-29 & 1.649 & 51,5 \\
\hline $30-39$ & 975 & 30,5 \\
\hline$\geq 40$ & 111 & 3,5 \\
\hline \multicolumn{3}{|l|}{ Escolaridade materna (anos) $(n=3.004)$} \\
\hline $0-4$ & 278 & 9,3 \\
\hline $5-8$ & 924 & 30,7 \\
\hline$\geq 9$ & 1.802 & 60,0 \\
\hline \multicolumn{3}{|l|}{ Raça/cor da pele da mãe $(n=3.186)$} \\
\hline Branca & 463 & 14,5 \\
\hline Parda & 2.402 & 75,4 \\
\hline Preta & 321 & 10,1 \\
\hline \multicolumn{3}{|c|}{ Mãe vive com marido ou companheiro ( $n=3.241$ ) } \\
\hline Não & 1.198 & 37,0 \\
\hline Sim & 2.043 & 63,0 \\
\hline Total & 3.242 & 100 \\
\hline
\end{tabular}


Tabela 2 - Distribuição de crianças com Caderneta de Saúde da Criança ou outro registro de vacinação, na linha de base (TO) e no primeiro acompanhamento (T1) do estudo Avaliação do Impacto do Programa Criança Feliz, Brasil (TO, agosto/2018-abril/2019; T1, setembro/2019-janeiro/2020)

\begin{tabular}{|c|c|c|}
\hline \multirow{2}{*}{ Variável } & $\begin{array}{l}\text { n (\%) com caderneta ou outro } \\
\text { registro de vacina }(\mathrm{TO})\end{array}$ & $\begin{array}{l}\text { n (\%) com caderneta ou outro } \\
\text { registro de vacina }{ }^{\mathrm{a}} \text { (T1) }\end{array}$ \\
\hline & $(n=3.239)$ & $(n=3.008)$ \\
\hline Nível socioeconômico da família (quintis) & $p=0,420^{b}$ & $\mathrm{p}=0,152^{\mathrm{b}}$ \\
\hline $1^{0}$ (mais pobre) & $631(97,5)$ & $554(91,7)$ \\
\hline $2^{\circ}$ & $627(96,8)$ & $564(91,6)$ \\
\hline $3^{\circ}$ & $629(97,1)$ & $553(91,3)$ \\
\hline $4^{0}$ & $618(95,4)$ & $568(94,2)$ \\
\hline $5^{\circ}$ (mais rico) & $625(96,9)$ & $537(90,3)$ \\
\hline Idade materna (anos) & $p=0,257^{b}$ & $p=0,103^{b}$ \\
\hline$<20$ & $445(95,9)$ & $389(90,6)$ \\
\hline $20-29$ & $1.596(96,6)$ & $1.396(91,1)$ \\
\hline 30-39 & $946(97,1)$ & $854(93,4)$ \\
\hline$\geq 40$ & $110(99,1)$ & $103(95,1)$ \\
\hline Escolaridade materna (anos) & $\mathrm{p}=0,199^{\mathrm{b}}$ & $\mathrm{p}=0,131^{\mathrm{b}}$ \\
\hline $0-4$ & $273(98,2)$ & $249(94,8)$ \\
\hline $5-8$ & $901(97,5)$ & $772(92,6)$ \\
\hline$\geq 9$ & $1.737(96,5)$ & $1.557(91,3)$ \\
\hline Raça/cor da pele da mãe & $p=0,493^{b}$ & $\mathrm{p}=0,322^{\mathrm{b}}$ \\
\hline Branca & $447(96,5)$ & $387(90,5)$ \\
\hline Parda & $2.326(97,0)$ & $2.068(93,7)$ \\
\hline Preta & $311(96,9)$ & $275(91,8)$ \\
\hline Mãe vive com marido ou companheiro & $\mathrm{p}=0,094^{\mathrm{b}}$ & $\mathrm{p}=0,012^{\mathrm{b}}$ \\
\hline Não & $1.149(96,0)$ & $1.016(90,1)$ \\
\hline Sim & $1.983(97,2)$ & $1.762(92,8)$ \\
\hline Total & $3.133(96,7)$ & $2.779(92,4)$ \\
\hline
\end{tabular}

a) Proporções calculadas considerando-se os níveis hierárquicos do modelo multinível (nível 3, estado; nível 2, município; nível 1, crianças); b) Teste de Wald.

Criança ou outro registro de vacinas aplicadas, no momento da entrevista. No T0, não houve diferença entre as crianças cujas mães apresentaram a caderneta ou outro registro de vacinação, de acordo com qualquer das variáveis independentes analisadas (Tabela 2). No T1, entretanto, foi maior a proporção de crianças com caderneta ou registro de vacinação filhas de mães que viviam com marido ou companheiro, em comparação a suas contrapartes (Tabela 2).

A proporção de crianças com vacinação adequada foi 2,5 vezes maior no $\mathrm{T} 0\left(61,0 \%-\mathrm{IC}_{95 \%} 59,3 ; 62,6\right)$, em relação ao T1 $\left(24,8 \%\right.$ - $\left.\mathrm{IC}_{95 \%} 22,8 ; 25,9\right)(\mathrm{p}<0,001)$. $\mathrm{Na}$ Tabela 3, são apresentadas as proporções de crianças com vacinação adequada, de acordo com características da família e maternas. No T0, a maior proporção de vacinação adequada ocorreu entre as pertencentes ao quintil mais rico $(67,9 \%)$ e entre filhos de mães com $\geq 9$ anos de escolaridade (63,3\%) (Tabela 3). No T1, não foram observadas diferenças entre as proporções de vacinação adequada, conforme as variáveis independentes.

Na Tabela 4 são apresentados os percentuais de vacinação adequada, conforme o calendário vacinal e a idade das crianças em meses. Não se evidenciou um padrão de aumento ou redução da cobertura conforme a idade. No primeiro acompanhamento (T0), a faixa 
Tabela 3 - Proporção de crianças com vacinação adequada conforme calendário vacinal com 15 dias de tolerância (T0 e T1), de acordo com características familiares e maternas na linha de base do estudo Avaliação do Impacto do Programa Criança Feliz, Brasil (TO, agosto/2018-abril/2019; T1, setembro/2019-janeiro/2020)

\begin{tabular}{|c|c|c|}
\hline \multirow{4}{*}{ Características familiares e maternas } & TO & T1 \\
\hline & $\begin{array}{l}\text { Vacinação adequada conforme calendário } \\
\text { vacinal com } 15 \text { dias de tolerância }{ }^{a}\end{array}$ & $\begin{array}{l}\text { Vacinação adequada conforme calendário } \\
\text { vacinal com } 15 \text { dias de tolerância }{ }^{a}\end{array}$ \\
\hline & $(n=3.205)$ & $(n=3.008)$ \\
\hline & n (\%) & n (\%) \\
\hline Nível socioeconômico da família (quintis) & $\mathrm{p}<0,001^{\mathrm{b}}$ & $\mathrm{p}=0,557^{\mathrm{b}}$ \\
\hline $1^{\circ}$ (mais pobre) & $315(55,1)$ & $164(26,6)$ \\
\hline $2^{0}$ & $396(57,6)$ & $145(22,7)$ \\
\hline $3^{0}$ & $374(58,4)$ & $149(24,5)$ \\
\hline $4^{0}$ & $420(63,1)$ & $144(24,3)$ \\
\hline $5^{\circ}$ (mais rico) & $447(67,9)$ & $143(26,0)$ \\
\hline Idade materna (anos) & $p=0,341^{b}$ & $p=0,474^{b}$ \\
\hline$<20$ & $273(61,6)$ & $107(25,2)$ \\
\hline 20-29 & $959(59,3)$ & $362(23,6)$ \\
\hline $30-39$ & $633(62,4)$ & $238(26,3)$ \\
\hline$\geq 40$ & $71(63,2)$ & $28(26,6)$ \\
\hline Escolaridade materna (anos) & $p=0,001^{b}$ & $\mathrm{p}=0,780^{b}$ \\
\hline $0-4$ & $157(56,3)$ & $65(24,1)$ \\
\hline $5-8$ & $521(57,2)$ & $202(24,0)$ \\
\hline$\geq 9$ & $1.125(63,3)$ & $419(25,2)$ \\
\hline Raça/cor da pele da mãe & $p=0,573^{b}$ & $p=0,797^{b}$ \\
\hline Branca & $293(60,2)$ & $95(23,6)$ \\
\hline Parda & $1.438(60,4)$ & $571(24,1)$ \\
\hline Preta & $196(63,3)$ & $65(25,0)$ \\
\hline Mãe vive com marido ou companheiro & $\mathrm{p}=0,899^{\mathrm{b}}$ & $\mathrm{p}=0,092^{\mathrm{b}}$ \\
\hline Não & $716(60,4)$ & $258(23,1)$ \\
\hline Sim & $1.238(60,6)$ & $488(25,8)$ \\
\hline Total & $1.954(61,0)$ & $746(24,8)$ \\
\hline
\end{tabular}

a) Proporções calculadas considerando-se os níveis hierárquicos do modelo multinível (nível 3, estado; nível 2, município; nível 1, crianças); b) Teste de Wald.

de idade com menor percentual de vacinas adequadas correspondeu ao estrato de 5,5 a 6,5 meses (53,5\%); e 0 maior percentual, ao estrato de 0,5 a 2,5 meses (93,5\%). No segundo acompanhamento (T1), o maior percentual de vacinas adequadas correspondeu à faixa de idade de 12,5 a $15,5(34,4 \%)$ meses, e 0 menor, à faixa dos 6,5 aos $9,5(12,5 \%)$ meses.

Os Materiais Suplementares 1 e 2 apresentam os percentuais de vacinação adequada, respectivamente para cada UF e município estudado. A maior proporção de vacinação adequada no T0 foi observada no
Ceará (78,4\%), e a menor, no Pará (36,1\%) (Material Suplementar 1). Segundo observado no T1, não foram observadas diferenças significativas entre as UFs. Das 30 cidades estudadas, Morada Nova (CE) teve a maior cobertura vacinal no T0 (89,3\%), e São Miguel do Guamá (PA), a maior cobertura vacinal no T1 (45,7\%) (Material Suplementar 2). A menor cobertura vacinal no T0 foi verificada em Tailândia (PA), com 10,6\%; e no T1, em Paulo Afonso (BA), com 7,2\%.

Segundo cada UF, entre os municípios estudados, os maiores percentuais de vacinação no T0 foram 
Tabela 4 - Percentuais de vacinação adequada conforme calendário vacinal com 15 dias de tolerância, de acordo com a idade da criança em meses, na linha de base (TO) e no primeiro acompanhamento (T1) do estudo Avaliação do Impacto do Programa Criança Feliz, Brasil (TO, agosto/2018-abril/2019; T1, setembro/2019-janeiro/2020)

\begin{tabular}{lccc}
\hline \multirow{2}{*}{ Idade em meses } & Percentual de vacinas adequadas no To & & Percentual de vacinas adequadas no T1 \\
\cline { 2 - 3 } $\mathbf{n n} 2,5-2,5$ & $\mathbf{n}(\%)$ & $\mathbf{n}(\%)$ \\
$2,5-3,5$ & $878 / 201(93,5)$ & - \\
$3,5-4,5$ & $128 / 123(70,7)$ & - \\
$4,5-5,5$ & $146 / 272(56,0)$ & - \\
$5,5-6,5$ & $192 / 359(53,5)$ & - \\
$6,5-9,5$ & $667 / 1217(54,8)$ & $1 / 8(12,5)$ \\
$9,5-12,5$ & $546 / 832(65,6)$ & $36 / 122(29,5)$ \\
$12,5-15,5$ & $0 / 7(0,0)$ & $177 / 514(34,4)$ \\
$15,5-26,5$ & - & $532 / 2.364(22,5)$ \\
\hline
\end{tabular}

encontrados em Irecê (BA), Morada Nova (CE), Novo Gama (G0), São Miguel do Guamá (PA), Serra Talhada (PE) e Piracicaba (SP); e os menores percentuais, nos municípios de Serrinha (BA), Caucaia (CE), Águas Lindas de Goiás (G0), Tailândia (PA), Abreu e Lima (PE) e Francisco Morato (SP). No T1, os municípios com maiores percentuais de vacinação adequada foram: Irecê e Vitória da Conquista (BA), Sobral (CE), Águas Lindas de Goiás (G0), São Miguel do Guamá (PA), Caruaru (PE) e Piracicaba (SP); e os municípios com menores percentuais, Paulo Afonso (BA), Caucaia (CE), Novo Gama (G0), Bragança (PA), São Lourenço da Mata (PE) e Limeira (SP) (Material Suplementar 2).

A proporção de crianças que receberam cada uma das doses de vacina no T0 e no T1, de acordo com 0 calendário vacinal para a idade, é apresentada no Material Suplementar 3. No T0, a dose vacinal com menor cobertura foi 0 reforço da vacina meningocócica $\mathrm{C}$ (14,3\%). No T1, a menor proporção correspondeu à dose do primeiro reforço da DTP $(6,1 \%)$.

\section{Discussão}

Os resultados deste estudo revelaram baixo percentual de crianças com vacinação adequada, tanto no primeiro quanto no segundo ano de vida, mesmo respeitando-se os 15 dias de tolerância em relação à faixa etária recomendada para a aplicação da vacina. Os percentuais de vacinação adequada no primeiro ano de vida foram maiores entre crianças pertencentes a famílias do quintil mais rico e cujas mães tinham $\geq 9$ de escolaridade. Essas diferenças socioeconômicas devem ser interpretadas levando-se em conta a constituição da amostra do estudo: famílias de menor nível socioeconômico, beneficiárias do Bolsa Família. No segundo acompanhamento (T1), não se encontrou relação entre cobertura vacinal e nível socioeconômico ou escolaridade materna, o que, possivelmente, é reflexo da diminuição da oferta de vacinas no ano de 2019. ${ }^{18}$

Estudo realizado no município de Volta Redonda, RJ, com crianças de 2 meses a 5 anos de idade atendidas em unidades básicas de saúde (UBS), encontrou cobertura vacinal completa de apenas $11 \%$ no ano de 2012. ${ }^{19}$ Já outro estudo, realizado no âmbito do programa das Coortes de Nascimentos de Pelotas, RS, revelou que, aos 12 meses de vida, a cobertura vacinal foi de $80,9 \%\left(\mathrm{IC}_{95 \%} 79,8 ; 82,0\right), 97,2 \%\left(\mathrm{IC}_{95 \%}, 96,1 ; 98,0\right), 87,8 \%$ $\left(\mathrm{IC}_{95 \%} 86,7 ; 88,8\right)$ e $77,2 \%\left(\mathrm{IC}_{95 \%} 75,8 ; 78,4\right)$, respectivamente, para nascidos no município gaúcho em 1982, 1993, 2004 e 2015. ${ }^{6}$

Resultados de inquéritos populacionais, a exemplo estudos mencionados em epígrafe e desta pesquisa, tendem a mostrar coberturas vacinais inferiores àquelas obtidas da rotina dos sistemas de informações de saúde. Estes sistemas são alimentados por relatórios de doses aplicadas, compilados nas unidades sanitárias. Os cálculos de cobertura utilizam-se de estimativas populacionais como denominadores - o que, às vezes, resulta em coberturas superiores a $100 \%$. Por exemplo, segundo dados da série Saúde Brasil, ${ }^{20}$ em 2015, a cobertura da vacina do bacilo de Calmette-Guérin (BCG) 
foi de $105 \%{ }^{20}$ No ano de 2016 , as doses da vacina da hepatite $B(<1$ ano), rotavírus humano $(<1$ ano), meningocócica $\mathrm{C}$ (12 meses), vacina absorvida difteria, tétano epertussis (DTP) (15 meses), pneumocócica 10 (12 meses), poliomielite (15 meses) e tríplice viral (12 meses) aplicadas estiveram, todas, abaixo da meta de cobertura vacinal. ${ }^{21} \mathrm{~A}$ despeito dos diferentes métodos de cálculo empregados, os dados oficiais confirmam queda nas coberturas em nível nacional.

0 atual estudo demonstrou que pertencer ao quintil mais rico - no âmbito de uma amostra predominantemente pobre - foi um fator associado a maiores proporções de vacinação adequada no T0. Dois estudos avaliativos, sobre países de renda média e baixa, verificaram desigualdades na cobertura vacinal conforme 0 nível socioeconômico: cobertura menor para o quintil mais pobre, quando comparada à cobertura do quintil mais rico. ${ }^{22,23}$

Dois estudos brasileiros, realizados em Salvador, $\mathrm{BA},{ }^{24}$ entre 2007 e 2008, e em São Luís, MA, ${ }^{25}$ entre 2006 e 2007, apontaram que crianças dos estratos sociais mais pobres tiveram menor cobertura vacinal, comparadas àquelas dos estratos mais ricos. De sua parte, os estudos sobre as coortes de nascimentos de Pelotas observaram que, aos 12 meses de idade, houve maior cobertura vacinal entre as criança nascidas dos estratos mais ricos nascidas em 1982, enquanto, para as nascidas em 2015, tal padrão socioeconômico havia-se invertido, fato que seus autores atribuíram à possível existência de uma hesitação das famílias mais ricas em vacinar seus filhos. ${ }^{6}$ Ainda segundo os autores da série histórica, outro motivo importante para a queda da cobertura vacinal diz respeito à complexidade crescente do esquema vacinal brasileiro, que passou de quatro tipos de vacinas em 1982, administradas em cinco contatos com os serviços durante o primeiro ano de vida, para 18 tipos de vacinas em oito contatos. Se, por um lado, a expansão na oferta de vacinas é importante para o controle de doenças imunopreveníveis, por outro lado, a complexidade dos esquemas contribui para a redução da cobertura prevista no calendário vacinal.

A menor escolaridade materna associou-se a menores coberturas no T0. 0 estudo realizado em UBS de Volta Redonda, RJ, encontrou maiores prevalências de crianças com vacinação em atraso entre filhos de mães com menos de oito anos de estudo. ${ }^{19}$ Outro estudo, conduzido no Maranhão entre 2006 e 2007, com crianças de 12 a 59 meses de vida, revelou maiores prevalências de esquema vacinal incompleto entre famílias cujo chefe de família tinha menos de cinco anos de escolaridade. ${ }^{25}$

De modo geral, destacam-se como pontos fortes do presente estudo (i) o tamanho e a abrangência da amostra, envolvendo mais de 3 mil crianças de seis Unidades da Federação brasileira e 30 municípios de quatro das cinco macrorregiões do país, e (ii) a estratégia adotada para coleta da informação sobre o desfecho, incluindo a fotografia das Cadernetas de Saúde da Criança e a extração direta do registro vacinal. Muitos estudos utilizam apenas a informação relatada pelas mães ou guardiões sobre as vacinas recebidas pela criança. A análise dos dados retirados de dois acompanhamentos - T0 e T1 - permitiu comparar a cobertura vacinal na mesma população, no período de um ano.

Entretanto, a pesquisa apresenta uma limitação: o fato de utilizar dados de uma população predominantemente pobre, originária de um estudo planejado para responder uma outra questão de pesquisa, limita a generalização de seus resultados. Informações sobre uso de serviços de saúde pelas crianças e disponibilidade de vacinas para elas nas unidades básicas do Sistema Único de Saúde não puderam ser avaliados.

As diferenças nas prevalências de cobertura vacinal entre o primeiro e o segundo acompanhamentos permitem considerar a seguinte hipótese: parte da diminuição na cobertura no período seria reflexo da falta de vacinas, principalmente da pentavalente em $2019 .{ }^{18}$ Também chamam a atenção as amplas diferenças nas coberturas vacinais entre UFs e municípios. Essas diferenças resultam, de um lado, das características locais da implementação do PNI (oferta de vacinas, acesso aos serviços de saúde e regularidade do registro na Caderneta de Saúde da Criança), e de outro lado, dependem da adesão das famílias à vacinação de suas crianças. Como a vacinação constitui uma das condicionalidades para 0 recebimento dos benefícios do Bolsa Família, resta mais forte a hipótese de essas diferenças atribuírem-se a características locais de implementação do programa. Diferenças semelhantes foram identificadas em 2017, quando o percentual de crianças até 2 anos vacinadas foi de $24,7 \%$ na Bahia, 25,7\% no Ceará, 23,4\% em Goiás, 42,1\% no Pará, 33\% em Pernambuco e 15,8\% em São Paulo. ${ }^{26}$

Destaca-se, outrossim, a baixa cobertura vacinal, ainda que se respeite uma tolerância de 15 dias de 
atraso para cada vacina. Assim, torna-se preocupante 0 fato de todas as crianças avaliadas pertencerem a famílias dependentes dos benefícios do Bolsa Família, cujo acesso está condicionado à vacinação de todos os menores de 7 anos na família. ${ }^{27}$ os achados do estudo sugerem a necessidade de uma avaliação detalhada da adesão das famílias à condicionalidade de manter 0 esquema de vacinação de suas crianças em dia.

Programas de visitas domiciliares podem aumentar a cobertura vacinal em crianças. ${ }^{28}$ No presente estudo, no T1, a cobertura vacinal no grupo PCF foi maior do que no controle, mesmo após ajuste para o status 'intervenção' ou 'controle', indicando que o Programa Criança Feliz pode contribuir para a melhoria das taxas de vacinação.

0 Ministério da Saúde, enquanto instituição responsável, tem demonstrado preocupação com o declínio das coberturas vacinais no país. A Organização Mundial da Saúde recomenda coberturas de 90\% para BCG e rotavírus humano, e de $95 \%$ para os demais imunizantes. As medidas a serem tomadas para promover a vacinação incluem (i) ampliar o horário dos locais de vacinação, (ii) evitar barreiras de acesso, (iii) aproveitar oportunidades para a vacinação (consultas ou outros procedimentos nas unidades de saúde), (iv) identificar crianças em atraso no calendário

\section{Referências}

1. Andre FE, Booy R, Bock HL, Clemens J, Datta SK, John TJ, et al. Vaccination greatly reduces disease, disability, death and inequity worldwide. Bull World Health Organ. 2008;86(2):81-160. doi: https://doi.org/10.2471/blt.07.040089.

2. World Health Organization. Immunization [Internet]. [Geneva]: World Health Organization; 2019 [acesso 20 jul. 2020]. Disponível em: https://www.who.int/ news-room/facts-in-pictures/detail/immunization

3. Ministério da Saúde (BR). Manual de normas e procedimentos para vacinação [Internet]. Brasília, DF: MS; 2014 [acesso 17 jun. 2020]. Disponível em: https://bvsms.saude.gov.br/bvs/publicacoes/ manual_procedimentos_vacinacao.pdf

4. Ministério da Saúde (BR). Programa Nacional de Imunização: coberturas vacinais no Brasil, período: 2010 - 2014 [Internet]. Brasília, DF: MS; 2015 [acesso 17 jun. 2020]. Disponível em: https://portalarquivos2. vacinal, mediante busca ativa e estratégias comunitárias, (v) promover ações coletivas de educação em saúde, junto com a comunidade, para a prevenção de doenças por meio da vacinação, e (vi) combater qualquer informação falsa sobre vacinação, sempre exaltando a segurança e benefícios das vacinas. ${ }^{29}$

Cumpre salientar que, tão logo se concluiu a pesquisa em tela, a pandemia da COVID-19 chegou ao país e, muito provavelmente, relatos mais recentes, informando que ações preventivas na rede básica foram severamente afetadas pela pandemia, sinalizam para uma cobertura vacinal - até o final de 2020 - todavia menor do que a evidenciada na presente análise.

\section{Contribuição dos autores}

Barcelos RS, Santos IS, Munhoz TN, Blumenberg C, Bortolotto CC, Matijasevich A, Salum C, Santos Júnior HG, Marques L, Correia L, Souza MR, Lira PIC, Altafim E, Macana EC e Victora CG contribuíram com a concepção e desenho do trabalho, coleta, análise e interpretação dos dados, redação e revisão crítica de conteúdo intelectual do manuscrito. Todos os autores aprovaram a versão final do manuscrito e são responsáveis por todos os seus aspectos, incluindo a garantia de sua precisão e integridade.

saude.gov.br/images/pdf/2017/agosto/17/ AACOBERTURAS-VACINAIS-NO-BRASIL---2010-2014.pdf

5. Domingues CMAS, Teixeira AMS. Coberturas vacinais e doenças imunopreveníveis no Brasil no período 1982-2012: avanços e desafios do Programa Nacional de Imunizações. Epidemiol Serv Saude. 2013;22(1):9-27. doi: http://dx.doi. org/10.5123/S1679-49742013000100002.

6. Silveira MF, Buffarini R, Bertoldi AD, Santos IS, Barros AJD, Matijasevich A, et al. The emergence of vaccine hesitancy among upper-class Brazilians: results from four birth cohorts, 1982-2015. Vaccine. 2020 jan 16;38(3):482-8. doi: http:// dx.doi.org/10.1016/j.vaccine.2019.10.070.

7. Ministério do Desenvolvimento Social e Agrário (BR). A intersetoralidade na visita domiciliar [Internet]. Brasília, DF: MDS; 2017 [acesso 23 dez. 2020]. (Programa criança feliz). Disponível em: 
http://www.mds.gov.br/webarquivos/publicacao/crianca_ feliz/A_intersetorialidade_na_visita_domiciliar_2.pdf

8. Ministério da Cidadania (BR). Criança feliz: quanto mais cuidado, mais futuro [Internet]. Brasília DF; 2017 [acesso 23 dez. 2020]. Disponível em: http:// cidadania.gov.br/criancafeliz/campanha/

9. Santos IS, Munhoz TN, Barcelos RS, Blumenberg C, Bortolotto CC, Matijasevich A, et al. Estudo de Linha de Base da Avaliação de Impacto do Programa Criança Feliz. Cad Estud. 2020;(35):13-31.

10. Victora C, Santos I, Munhoz T. Avaliação do Programa Criança Feliz: projeto de pesquisa [Internet]. Pelotas, RS: Programa de Pós-graduação em Epidemiologia; 2020 [acesso 21 jun. 2020]. Disponível em: http://www. epidemio-ufpel.org.br/uploads/downloads/avaliacaodo-impacto-do-programa-crianca-feliz.pdf Disponível

11. Multiple Indicator Cluster Surveys. MICS6 Questionnaires [Internet]. UNICEF; 2017 [acesso 05 mai. 2020]. Disponível em: http://mics.unicef.org/tools

12. Santos IS, Barros AJD, Matijasevich A, Domingues MR, Barros FC, Victora CG. Cohort profile: the 2004 Pelotas (Brazil) birth cohort study. Int J Epidemiol. 2011;40(6):1461-8. doi: http://dx.doi.org/10.1093/ije/dyq130.

13. Hallal PC, Bertoldi AD, Domingues MR, Silveira MF, Demarco FF, Silva ICM, et al. Cohort Profile: The 2015 Pelotas (Brazil) Birth Cohort Study. Int J Epidemiol. 2018 Aug 1;47(4):1048-1048h. doi: http://dx.doi.org/10.1093/ije/dyx219.

14. Ministério do Desenvolvimento Social (BR). Guia para visita domiciliar [Internet]. Brasília, DF: MDS; 2020 [acesso 10 mar. 2020]. (Programa crianca feliz). Disponível em: http://www.mds.gov. br/webarquivos/arquivo/crianca_feliz/Guia\%20 para\%20Visita\%20Domiciliar\%20-\%20Programa\%20 Crian\%C3\%A7a\%20Feliz\%20-\%2021-06-2017.pdf

15. Harris PA, Taylor R, Thielke R, Payne J, Gonzalez N, Conde JG. Research electronic data capture (REDCap)- a metadata-driven methodology and workflow process for providing translational research informatics support. J Biomed Inform. 2009;42(2):377-81.

16. Ministério da Saúde (BR). Vacinação [Internet]. Brasília, DF: MS; 2020 [acesso 23 mai. 2020]. Disponível em: http://www. saude.gov.br/saude-de-a-z/vacinacao
17. Ministério da Saúde (BR). Municípios conforme áreas de recomendação para vacinação contra febre amarela [Internet]. Brasília, DF: MS; 2017 [acesso 20 jun. 2020]. Disponível em: http:// portalarquivos.saude.gov.br/images/pdf/2017/abril/07/ Municipios-conforme-Areas-de-recomendacaopara-vacinacao-contra-febre-amarela.pdf

18. Brasil. Postos de saúde são reabastecidos com vacina pentavalente 2020 [Internet]. Brasília, DF; 2020 [acesso 27 dez. 2020]. Disponível em: https:/www.gov.br/pt-br/ noticias/saude-e-vigilancia-sanitaria/2020/01/postosde-saude-sao-reabatecidos-com-vacina-pentavalente

19. Cardoso MDT, Carneiro SG, Ribeiro TT, Strapasson JF, Carneiro RG. Avaliação da cobertura vacinal em crianças de 2 meses a 5 anos na estratégia saúde da família. Rev APS. 2015;18(3):273-80.

20. Ministério da Saúde (BR). Saúde Brasil, 2019: Uma análise da situação de saúde com enfoque nas doenças imunopreveníveis e na imunização [Internet]. Brasília, DF: MS; 2019 [acesso 02 jul. 2020]. Disponível em: https:/www.saude.gov.br/images/pdf/2019/ dezembro/05/Saude-Brasil-2019-imunizacao.pdf

21. Cruz A. A queda da imunização no Brasil. Rev Consensus [Internet]. 2017 [acesso 6 jun. 2020];(25):20-9. Disponível em: https://portal. fiocruz.br/sites/portal.fiocruz.br/files/documentos/ revistaconsensus_25_a_queda_da_imunizacao.pdf

22. Restrepo-Méndez MC, Barros AJD, Wong KL, Johnson HL, Pariyo G, França G, et al. Inequalities in full immunization coverage: trends in low- and middleincome countries. Bull World Health Organ. 2016 nov 1;94(11):794-805B. doi: http://dx.doi.org/10.2471/BLT.15.162172.

23. Hosseinpoor AR, Bergen N, Schlotheuber A, Gacic-Dobo M, Hansen PM, Senouci K, et al. State of inequality in diphtheria-tetanus-pertussis immunisation coverage in low-income and middle-income countries: a multicountry study of household health surveys. Lancet Glob Health. 2016;4(9):e617-26. doi: http:// dx.doi.org/10.1016/S2214-109X(16)30141-3

24. Barata RB, Pereira SM. Desigualdades sociais e cobertura vacinal na cidade de Salvador, Bahia. Rev Bras Epidemiol. 2013;16(2):266-77. doi: http:// dx.doi.org/10.1590/S1415-790X2013000200004.

25. Yokokura AVCP, Silva AAM, Bernardes ACF, Lamy FF, Alves MTSSB, Cabral NAL, et al. Cobertura 
vacinal e fatores assovciados ao esquema vacinal básico incompleto aos 12 meses de idade, São Luís, Maranhão, Brasil, 2006. Cad Saude Publica. 2006;29(3):522-34. doi: http://dx.doi. org/10.1590/S0102-311X2013000300010.

26. Ministério da Saúde (BR). Sistema de Informação do Programa Nacional de Imunizações: 2020 [Internet]. Brasília, DF; 2020 [acesso 22 dez. 2020]. Disponível em: http://sipni.datasus.gov.br/si-pni-web/faces/inicio.jsf

27. Ministério da Cidadania (BR). Bolsa família e cadastro único no seu município: informações detalhadas sobre todos os municípios do país [Internet]. Brasília, DF: Ministério da Cidadania; 2019 [acesso
02 jul. 2020]. Disponível em: https://www.gov.br/ cidadania/pt-br/acoes-e-programas/bolsa-familia

28. Isaac MR, Chartier M, Brownell M, Chateau D, Nickel NC, Martens P, et al. Can opportunities be enhanced for vaccinating children in home visiting programs? A population-based cohort study. BMC Public Health. 2015 jul 7;15:620. doi: http://dx.doi.org/10.1186/s12889-015-1926-8.

29. Conselho Nacional de Secretarias Municipais de Saúde (BR). Dez passos para ampliar a cobertura vacinal [Internet]. Brasília, DF: CONASEMS; 8 out. 2019 [acesso 04 jun 2020]. Disponível em: https://www.conasems. org.br/dez-passos-para-ampliar-cobertura-vacinal/

\section{Abstract}

Objective: To assess vaccination coverage, based on the National Immunization Program schedule, among children receiving financial support from the Family Income Transfer Program, Brazil, according to the family socioeconomic status and maternal characteristics. Methods: 3,242 children under 12 months old were assessed between August/2018 and April/2019, of whom 3,008 were reassessed between September/2019 and January/2020. The analyses were performed using multilevel models (level 3, Federative Unit; level 2, municipality; level 1, children). Results: Vaccination coverage was 2.5fold higher in the first follow-up (61.0\% - 95\% CI 59.3;62.6\%), compared to the second followup (24.8\% - 95\% CI 22.8;25.9\%) ( $p<0.001)$. In the first follow-up, coverage was higher in the richest quintile (67.9\%) and in children whose mothers had $\geq 9$ years of schooling (63.3\%). In the second follow-up, there were no differences. The highest coverage occurred between 0.52.5 (93.5\%) and 12.5-15.5 months (34.4\%), respectively, first and second follow-ups. Conclusion: Low coverage was found, both in the first and second year of life.

Keywords: Vaccination Coverage; Child; Immunization; Longitudinal Studies.

\section{Resumen}

Objetivo: Evaluar la cobertura de vacunación según el Programa Nacional de Inmunizaciones, entre los niños beneficiarios del Programa Bolsa Familia, Brasil, según el nivel socioeconómico familiar y características maternas. Métodos: Analizamos 3.242 niños menores de 12 meses entre agosto/2018 y abril/2019 y se reevaluaron 3.008 entre septiembre/2019 y enero/2020. Se utilizaron modelos multinivel (nivel 3, estado; nivel 2, municipio; nivel 1, niños). Resultados: La cobertura fue 2,5 veces mayor en el primer seguimiento (61,0\% $\left.-I C_{95 \%} 59,3 ; 62,6 \%\right)$ que en el segundo $\left(24,8 \%-I C_{95 \%}\right.$ $22,8 ; 25,9 \%)(p<0,001)$. En el primer seguimiento, la cobertura fue mayor en el quintil más rico $(67,9 \%)$ $y$ entre aquellos cuyas madres tenían $\geq 9$ años de escolaridad (63,3\%). En el segundo seguimiento no bubo diferencias. La mayor cobertura ocurrió entre los 0,5-2,5 (93,5\%) y 12,5-15,5 meses (34,4\%), respectivamente, en el primero y el segundo seguimiento. Conclusión: Se encontró baja cobertura en el primero y en el segundo año de vida.

Palabras clave: Cobertura de Vacunación; Niño; Inmunización; Estudios Longitudinales.

Recebido em 06/11/2020

Aprovado em 01/03/2021 
Material Suplementar 1 - Proporção de crianças com vacinação adequada, por estado, conforme calendário vacinal com 15 dias de tolerância, na linha de base (TO) e no primeiro acompanhamento (T1) do estudo Avaliação do Impacto do Programa Criança Feliz, Brasil (TO, agosto/2018-abril/2019; T1, setembro/2019-janeiro/2020)

\begin{tabular}{|c|c|c|}
\hline \multirow{4}{*}{ Unidade da Federação } & TO & T1 \\
\hline & $\begin{array}{l}\text { Vacinação adequada conforme calendário } \\
\text { vacinal com } 15 \text { dias de tolerância }{ }^{a}\end{array}$ & $\begin{array}{l}\text { Vacinação adequada conforme calendário } \\
\text { vacinal com } 15 \text { dias de tolerância }\end{array}$ \\
\hline & $(n=3.205)$ & $(n=3.008)$ \\
\hline & n (\%) & n (\%) \\
\hline & $p=0,001^{b}$ & $p=0,549^{b}$ \\
\hline Bahia & $297(59,4)$ & $80(19,4)$ \\
\hline Ceará & $662(78,4)$ & $237(28,0)$ \\
\hline Goiás & $176(57,6)$ & $79(28,8)$ \\
\hline Pará & $200(36,1)$ & $138(27,1)$ \\
\hline Pernambuco & $351(62,6)$ & $136(24,5)$ \\
\hline São Paulo & $268(67,8)$ & $76(21,8)$ \\
\hline Total & $1.954(61,0)$ & $746(24,8)$ \\
\hline
\end{tabular}

a) Proporções calculadas considerando-se os níveis hierárquicos do modelo multinível (nível 3, estado; nível 2, município; nível 1, crianças); b) Teste de Wald.

Material Suplementar 2 - Proporção de crianças com vacinas completas para idade até 15 dias após a idade exata, por município, na linha de base (TO) e no primeiro acompanhamento (T1) do estudo Avaliação do Impacto do Programa Criança Feliz, Brasil (T0, agosto/2018-abril/2019; T1, setembro/2019-janeiro/2020)

\begin{tabular}{|c|c|c|}
\hline \multirow{4}{*}{ Unidade da Federação/Município } & TO & T1 \\
\hline & $\begin{array}{l}\text { Vacinação adequada conforme calendário } \\
\text { vacinal com } 15 \text { dias de tolerância }\end{array}$ & $\begin{array}{l}\text { Vacinação adequada conforme calendário } \\
\text { vacinal com } 15 \text { dias de tolerância }\end{array}$ \\
\hline & $(n=3.205)$ & $(n=3.008)$ \\
\hline & n (\%) & $n(\%)$ \\
\hline \multicolumn{3}{|l|}{ Bahia } \\
\hline Casa Nova & $22(53,7)$ & $10(25,0)$ \\
\hline Feira de Santana & $122(55,5)$ & $22(10,9)$ \\
\hline Irecê & $40(88,9)$ & $13(28,9)$ \\
\hline Paulo Afonso & $52(46,0)$ & $7(7,2)$ \\
\hline Serrinha & $20(34,5)$ & $13(25,0)$ \\
\hline Vitória da Conquista & $41(77,4)$ & $15(28,9)$ \\
\hline \multicolumn{3}{|l|}{ Ceará } \\
\hline Caucaia & $83(60,1)$ & $23(15,9)$ \\
\hline Crato & $121(84,0)$ & $45(31,0)$ \\
\hline Itapipoca & $112(75,7)$ & $34(22,7)$ \\
\hline Juazeiro do Norte & $127(82,5)$ & $49(31,2)$ \\
\hline Morada Nova & $100(89,3)$ & $30(27,3)$ \\
\hline Sobral & $119(83,2)$ & $56(38,4)$ \\
\hline
\end{tabular}


Continuação

Material Suplementar 2 - Proporção de crianças com vacinas completas para idade até 15 dias após a idade exata, por município, na linha de base (TO) e no primeiro acompanhamento (T1) do estudo Avaliação do Impacto do Programa Criança Feliz, Brasil (T0, agosto/2018-abril/2019; T1, setembro/2019-janeiro/2020)

\begin{tabular}{|c|c|c|}
\hline \multirow{4}{*}{ Unidade da Federação/Município } & TO & T1 \\
\hline & $\begin{array}{l}\text { Vacinação adequada conforme calendário } \\
\text { vacinal com } 15 \text { dias de tolerância }\end{array}$ & $\begin{array}{l}\text { Vacinação adequada conforme calendário } \\
\text { vacinal com } 15 \text { dias de tolerância }\end{array}$ \\
\hline & $(n=3.205)$ & $(n=3.008)$ \\
\hline & n (\%) & n (\%) \\
\hline \multicolumn{3}{|l|}{ Goiás } \\
\hline Águas Lindas de Goiás & $56(45,9)$ & $36(34,6)$ \\
\hline Luziânia & $69(53,1)$ & $29(26,9)$ \\
\hline Novo Gama & $51(75,0)$ & $14(23,0)$ \\
\hline \multicolumn{3}{|l|}{ Pará } \\
\hline Altamira & $82(55,8)$ & $47(36,4)$ \\
\hline Bragança & $38(41,3)$ & $10(11,9)$ \\
\hline Breu Branco & $22(21,2)$ & $12(14,3)$ \\
\hline São Miguel do Guamá & $43(58,9)$ & $32(45,7)$ \\
\hline Tailândia & $15(10,6)$ & $37(29,4)$ \\
\hline \multicolumn{3}{|l|}{ Pernambuco } \\
\hline Abreu e Lima & $38(34,2)$ & $14(13,9)$ \\
\hline Camaragibe & $69(67,7)$ & $31(32,0)$ \\
\hline Caruaru & $94(79,0)$ & $44(37,9)$ \\
\hline São Lourenço da Mata & $53(52,0)$ & $9(9,0)$ \\
\hline Serra Talhada & $97(79,5)$ & $38(31,9)$ \\
\hline \multicolumn{3}{|l|}{ São Paulo } \\
\hline Francisco Morato & $69(57,5)$ & $23(20,2)$ \\
\hline Limeira & $76(69,1)$ & $17(16,8)$ \\
\hline Piracicaba & $34(82,9)$ & $11(28,2)$ \\
\hline Sumaré & $29(67,4)$ & $7(21,2)$ \\
\hline Taboão da Serra & $60(68,2)$ & $18(22,2)$ \\
\hline Total & $1.954(61,0)$ & $746(24,8)$ \\
\hline
\end{tabular}


Material Suplementar 3 - Percentual de doses de vacinas adequadas no estudo de linha de base (TO) e no primeiro ano de acompanhamento( $\mathrm{T} 1$ ) de acordo com o calendário vacinal e a idade recomendada, considerando-se 15 dias de tolerância, Brasil (TO, agosto/2018-abril/2019; T1, setembro/2019-janeiro/2020)

\begin{tabular}{|c|c|c|}
\hline \multirow{2}{*}{ Vacinas } & TO $(n=3.242)$ & $\mathrm{T} 1(\mathrm{n}=3.008)$ \\
\hline & n (\%) & n (\%) \\
\hline BCGa (dose única) & $189(94,0)$ & $189(94,0)$ \\
\hline Hepatite B (dose única) & $191(95,0)$ & $191(95,0)$ \\
\hline Pentavalente (1 $1^{\mathrm{a}}$ dose) & $99(80,5)$ & $99(80,5)$ \\
\hline VIPb (1 $1^{\mathrm{a}}$ dose) & $113(91,9)$ & $113(91,9)$ \\
\hline Pneumocócica 10-valente 10 ( $1^{\text {a }}$ dose) & $108(87,8)$ & $108(87,8)$ \\
\hline Rotavírus humano (1 dose) & $108(87,8)$ & $108(87,8)$ \\
\hline Meningocócica C ( $1^{\text {a }}$ dose) & $132(68,0)$ & $132(68,0)$ \\
\hline Pentavalente ( $2^{\mathrm{a}}$ dose) & $173(63,6)$ & $173(63,6)$ \\
\hline VIP $\left(2^{\mathrm{a}}\right.$ dose $)$ & $201(73,9)$ & $201(73,9)$ \\
\hline Pneumocócica 10-valente 10 ( $2^{\text {a }}$ dose) & $194(71,3)$ & $194(71,3)$ \\
\hline Rotavírus humano ( $2^{\mathrm{a}}$ dose) & $205(75,4)$ & $205(75,4)$ \\
\hline Meningocócica C ( $2^{\mathrm{a}}$ dose) & $209(58,2)$ & $209(58,2)$ \\
\hline Pentavalente ( $3^{\mathrm{a}}$ dose) & $173(44,3)$ & $173(44,3)$ \\
\hline VIPb (3a dose) & $202(51,7)$ & $202(51,7)$ \\
\hline Pneumocócica 10-valente (reforço) & $3(42,9)$ & $71(59,7)$ \\
\hline Meningocócica C (reforço) & $1(14,3)$ & $65(54,6)$ \\
\hline Tríplice viral (1ª dose) & $3(42,9)$ & $71(58,2)$ \\
\hline DTPc (10 reforço) & - & $12(6,1)$ \\
\hline VOPd (10 reforço) & - & $71(36,0)$ \\
\hline Hepatite A (dose única) & - & $71(36,0)$ \\
\hline Tetraviral (dose única) & - & $74(38,1)$ \\
\hline
\end{tabular}

a) BCG: bacilo de (almette-Guérin; b) VIP: poliomielite 1,2,3; c) DTP: vacina adsorvida difteria, tétano e pertussis; d) VOP: poliomielite 1 e 3. 


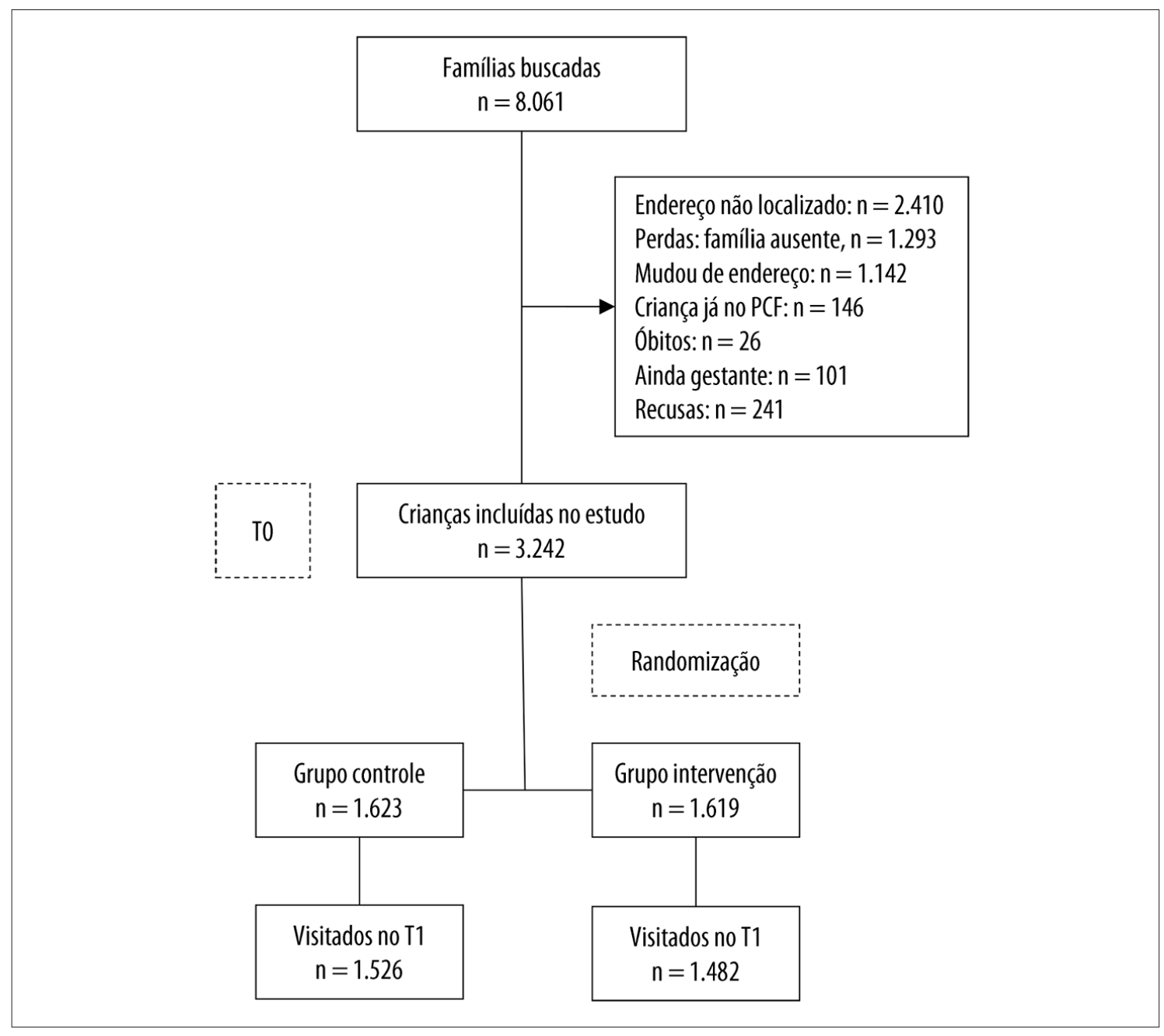

Material Suplementar 4 - Número de famílias buscadas e crianças incluídas no estudo 